\title{
ANÁLISE AMPLIADA DA CAPACIDADE NO ENLACE REVERSO DE UM SISTEMA CELULAR CDMA*
}

\author{
André Noll Barreto e João Célio Brandão \\ CETUC-PUC/Rio
}

\begin{abstract}
RESUMO: Neste trabalho apresentamos uma revisão do cálculo de capacidade no enlace reverso de sistemas CDMA baseados na padronizaçãso IS-95 introduzindo novos elementos no modelo do sistema e uma análise mais detalhada de alguns aspectos. A metodologia básica utilizada é aquela baseada na metodologia de Erlang para análise de tráfego aplicada em [1] para sistemas CDMA. O modelo utilizado em [1] é ampliado para levar em conta uma limitação no número de canais, associados ao número de receptores na estação rádio base. Além disto é apresentada uma análise detalhada do efeito de uma fila de espera para novas chamadas.
\end{abstract}

\begin{abstract}
In this paper, the calculation of the capacity in the reverse link of IS-95 CDMA cellular systems is reviewed with the introduction of new elements in the system model and a more detailed analysis of some aspects of the problem. The used methodology is the classical Erlang methodology for traffic analysis as applied in [1] to CDMA systems. The model presented in [1] is extended to include a limitation on the number of available channels, which correspond to the number of receivers in the base station. Besides that, a detailed analysis of the effect of a waiting queue for new calls is presented.
\end{abstract}

Palavras chaves: CDMA, Sistemas Celulares, Telefonia Celular

\section{INTRODUÇÃO}

A tecnologia CDMA para o sistema de acesso em telefonia móvel celular foi proposta recentemente, através da norma IS-95 [2] como solução competitiva na corrida pela implementação dos novos sistemas celulares digitais. Desde então, inúmeros estudos têm sido conduzidos no sentido de avaliar o desempenho desta tecnologia e compará-lo com o de outras tecnologias.

A referência [3] é a referência básica para avaliação de desempenho e capacidade de sistemas CDMA baseados no padrão IS-95. É feita uma análise para os enlaces direto e reverso concluindo-se que este último é o enlace mais crítico. As previsões de capacidade feitas em [3] podem ser consideradas otimistas e a conclusão numérica de que o sistema CDMA apresenta uma capacidade 18 vezes maior do que a oferecida pelo sistema AMPS é claramente exagerada.

Motivados pelas conclusões em [3], diversos trabalhos foram publicados posteriormente focalizando o cálculo de capacidade no enlace reverso em sistemas CDMA. Em [4,5] o modelo utilizado em [3] é avaliado através de simulação em computador e uma atenção especial é dada à modelagem de imperfeição no controle de potência. Verifica-se em [4] que uma flutuação de $1 \mathrm{~dB}$ na potência controlada leva a uma redução de $35 \%$ na capacidade. Além disto, é avaliada a sensibilidade da capacidade aos valores assumidos por outros parâmetros do modelo, calculando-se, por exemplo, uma redução de $20 \%$ ao se mudar o expoente da função de atenuação com a distância de 4 para 3 ou em $30 \%$ ao se mudar o fator de atividade da voz de $37,5 \%$ para $50 \%$.

A capacidade de um sistema de múltiplo acesso deve ser entendida como o máximo número de usuários que permita a operação dos enlaces com taxa de erro igual ou inferior a um valor máximo aceitável. Quando se considera o enlace reverso de um sistema homogêneo, controle de potência ideal e uma única célula, este cálculo fica bastante simplificado e pode ser feito a partir da expressão

$$
\frac{E_{b}}{N_{0}+(N-1) S / W} \geq \Gamma
$$

onde o lado esquerdo da desigualdade é a razão entre a energia média por bit e o dobro da densidade espectral de potência do ruído equivalente (soma das interferências e do ruído propriamente dito) e o lado direito é o valor mínimo aceitável desta razão (associada à taxa de erro máxima especificada). Explicitando-se $\mathrm{N}$ e desprezandose o ruído do receptor em presença das interferências, obtemos

$$
N_{\max } \cong \frac{W / R}{\Gamma}
$$




\section{André Noll Barreto e João Célio Brandão}

Esta expressão mostra a grande dependência da capacidade ao valor de $\Gamma$. Nos cálculos em [3] é utilizado o valor $\Gamma=5(7 \mathrm{~dB})$. No entanto se, devido a outras perturbações no canal, for necessária uma razão maior, a capacidade será sensivelmente reduzida. Diversos trabalhos $[6,7,8]$ procuram avaliar a taxa de erro em sistemas CDMA levando em conta as perturbações típicas do canal de comunicações móveis. Através destes estudos e de medidas de campo, pode-se chegar a um valor adequado de $\Gamma$ e este valor será então utilizado no cálculo da capacidade. Obviamente, em um sistema real, cada enlace poderá exigir um valor diferente de razão sinalruído mínima, de acordo com características específicas da área coberta. Porém, estes cenários específicos não se prestam a avaliações comparativas de caráter mais geral onde a homogeneidade é normalmente assumida.

Uma vez que os valores da razão sinal-ruído são considerados constantes, a capacidade também resultará em um número constante. Mas, ao se admitir variações aleatórias na razão sinal-ruído, a capacidade calculada através de (1) seria um número aleatório. Neste caso pode-se tomar a capacidade como uma capacidade média, ou como se faz em [3], associar a capacidade a uma probabilidade $\mathrm{p}_{0}$, de que a razão sinal-ruído seja inferior à mínima, caracterizando a queda do enlace. Ou seja, a capacidade seria calculada a partir da expressão

$$
P\left[\frac{E_{b}}{N_{0}^{T}} \leq \Gamma\right]=p_{0}
$$

onde $\mathrm{N}_{0}{ }^{\mathrm{T}}$ é a densidade espectral de potência do ruído equivalente. Em [3], na realidade, este cálculo só é introduzido ao se considerar a interferência de outras células, pois esta tem potência aleatória, mesmo com controle ideal de potência.

O estabelecimento de um critério probabilístico para desempenho do sistema celular nos remete à análise de tráfego pela metodologia de Erlang onde o critério básico de desempenho é a probabilidade de bloqueio e a flutuação do número de usuários no sistema é um elemento essencial do modelo. Trabalhos mais recentes de avaliação de capacidade passam a adotar este enfoque. embora, na prática acaba-se recaindo em (1) pois a probabilidade de bloqueio no sistema CDMA é associada à probabilidade de queda de enlace. Porém, o número de usuários $\mathrm{N}$ passa a ser agora o número (aleatório) de usuários no sistema em um dado momento e o que se procura obter é a média deste número, associada ao tráfego no sistema.

Em [1] esta metodologia é utilizada para se obter valores de tráfego associados a valores de probabilidade de bloqueio, considerando-se um modelo que se pretende bastante realista, considerando inclusive as flutuações no controle de potência e na própria razão sinal-ruído requerida. Este modelo tem se consolidado como um modelo de referência motivando contribuições recentes no sentido de ampliá-lo e aprimorá-lo $[9,10,11]$ No entanto, podemos observar que ainda há alguns aspectos que merecem tratamento mais preciso. Este é o caso do próprio critério básico de desempenho adotado. Como acabamos de comentar, a partir da hipótese de que não há bloqueio pela falta de recursos físicos (modems) o bloqueio é associado à probabilidade de queda de enlace. Embora possa ser uma boa aproximação, não só é possível, como também é interessante, considerar a limitação de canais como fator de bloqueio. Além disto, outros aspectos ainda podem ser considerados como o efeito de uma fila de espera no desempenho do sistema. A inclusão destes dois aspectos é a principal contribuição deste trabalho.

$\mathrm{O}$ artigo apresenta o seguinte organização: na seção 2, a seguir, fazemos uma revisão da análise apresentada em [1], na seção 3 introduzimos na análise a limitação do número de canais, na seção 4 analisamos o efeito de uma fila de espera no desempenho do sistema e na seção 5 apresentamos as principais conclusões do trabalho. $O$ trabalho contém também um Apêndice onde é analisada a relação entre a flutuação da interferência em um enlace e a perda de chamada.

\section{CAPACIDADE NO ENLACE REVERSO}

Com base em [1], apresentamos a seguir um resumo do cálculo da capacidade do enlace reverso de um sistema CDMA baseado no padrão IS-95. O modelo utilizado apresenta as características usuais, assim resumidas:

- um conjunto homogêneo de células de mesma dimensão com estações rádio bases situadas no centro

- um mesmo número de usuários por célula, todos transmitindo na mesma faixa de frequência, utilizando códigos quase ortogonais, de tal forma que o ganho de processamento é igual ao número de chips por bit.

- controle ideal de potência para usuários controlados pela mesma estação rádio base e ativação por voz

A metodologia de Erlang implica na suposição de um modelo de filas onde usuários entram e saem do sistema e podem ser bloqueados por falta de recursos físicos (canais de recepção e linhas de saída). Por outro lado, devido ao reuso universal de frequência no sistema CDMA, a variação do número de usuários no sistema introduz um novo fator de variabilidade na razão sinal-ruído no receptor de um usuário, ao lado dos fatores de propagação, controle de potência e ativação por voz.

Supondo que não há bloqueio pela falta de canais, a probabilidade de bloqueio é associada à queda de enlace, e esta se dá quando a razão sinal-ruído cai abaixo de um valor limiar. Assim, o principal objetivo da análise é determinar o comportamento aleatório da razão sinal ruído. 
Um comentário sobre o enfoque adotado em [1] é que, ao contrário de outros trabalhos [12] opta-se por se definir queda de enlace quando a densidade espectral de potência da interferência total for maior do que um certo valor $I_{0}$. $\mathrm{Na}$ realidade, o efeito da interferência no desempenho de um enlace depende, não do seu valor absoluto, mas sim do seu valor relativo à potência do sinal desejado. A análise com o valor absoluto da interferência será equivalente desde que se possa fixar o valor da potência do sinal desejado no receptor, o que corresponde a um controle ideal de potência para o sinal desejado. é preciso reconhecer que não têm sido discutidas em profundidade as implicações deste enfoque que apresenta como atrativo uma maior facilidade de análise probabilística. Ou seja, é mais simples determinar a distribuição probabilística da interferência do que da razão sinal-interferência.

Assumimos que cada célula tem um nível de tráfego igual a $\lambda / \mu$ Erlangs. Já que não existe um limite para o número de servidores, podemos modelar cada célula como uma fila $\mathbf{M} / \mathbf{M} / \infty$, e assim o número de usuários ativos $k$ por célula é uma variável aleatória de Poisson com média $\lambda \mu$. A condição para que não haja bloqueio é dada por

$$
\sum_{i=1}^{k} v_{i} E_{b_{i}} R+\sum_{j}^{\substack{\text { outras } \\ \text { celulas }}} \sum_{i=1}^{k} v_{i}^{(j)} E_{b i}^{(j)} R+N_{0} W \leq I_{0} W
$$

onde $E_{b i}^{(j)}$ é a energia por bit do $i$-ésimo usuário da $j$ ésima célula, e $v_{i}^{(j)}$ é uma variável aleatória de Bernoulli representando a atividade da voz do $i$-ésimo usuário da $j$ ésima célula, onde $\operatorname{Pr}(v=1)=\rho$. As variáveis $E_{b i}$ e $v_{i}$ se referem à célula alvo, para a qual está sendo feito o cálculo de desempenho.

Definindo-se $\varepsilon=E_{b} / I_{0}$, a condição dada em (4) pode ser rescrita da seguinte forma :

$$
Z \equiv \sum_{i=1}^{k} v_{i} \varepsilon_{i}+\sum_{j}^{\substack{\text { outras } \\ \text { celulas }}} \sum_{i=1}^{k} v_{i}^{(j)} \varepsilon_{i}^{(j)} \leq \frac{W}{R}(1-\eta)
$$

onde $\eta=N_{0} / I_{0} . \quad \mathrm{O}$ valor deste parâmetro está relacionado com flutuações na razão sinal-ruído em presença de tráfego reduzido (neste trabalho é usado, como em [1], o valor típico $\eta=0.1$ ). A probabilidade de queda de enlace pode ser expressa por

$$
P_{Q}=\operatorname{Pr}\left[Z>\frac{W}{R}(1-\eta)\right]
$$

Note-se que $Z$ é uma soma ponderada dos valores da razão sinal-ruído de cada usuário, dependendo portanto do número de usuários ativos. Além disso, como já foi comentado, se o controle de potência fosse ideal, a razão $E_{b} / I_{0}$ para cada usuário $\left(\varepsilon_{i}\right)$ seria constante e a mesma para todos os usuários. No entanto, em cada receptor pode haver imperfeição no ajuste da energia $E_{b}$ para o valor estabelecido, e também uma variação do valor estabelecido para se obter uma certa taxa de erro, de acordo com as condições de propagação. $O$ efeito conjunto destes dois fatores pode ser modelado por uma variável aleatória com distribuição log-normal com média e variância aproximadas respectivamente por $\mathrm{m}_{\mathrm{x}}=7 \mathrm{e}$ $\sigma_{\mathrm{x}}=2,5 \mathrm{~dB}[13]$.

Como $Z$ é a soma de $k$ variáveis aleatórias independentes e identicamente distribuídas, considerando que o número de usuários é grande o suficiente, foi realizado em [1] uma aproximação pelo teorema do limite central. Assim podese assumir para $Z$ uma distribuição gaussiana e o cálculo de probabilidade de bloqueio só dependerá de sua média $\mathrm{e}$ de sua variância. $\mathrm{Na}$ verdade, o número de usuários $\mathrm{k}$ também é uma variável aleatória, e para este caso não encontramos na literatura nada que pudesse justificar rigorosamente esta aproximação. Na prática, porém, os resultados obtidos por esta aproximação são bastante similares a resultados de simulações para níveis altos de tráfego.

Definindo $Z^{\prime}=Z e^{-\beta m_{x}}$ onde $\beta=0,1 \ln 10$ e utilizando a hipótese Gaussiana, podemos então escrever a seguinte expressão para a probabilidade de queda de enlace

$$
P_{Q} \approx \mathrm{Q}\left[\frac{A-\mathrm{E}\left(Z^{\prime}\right)}{\sqrt{\operatorname{Var}\left(\mathrm{Z}^{\prime}\right)}}\right]
$$

onde $\mathbf{Q}$ é a função erro modificada, dada por

$$
Q(\alpha)=\frac{1}{\sqrt{2 \pi}} \int_{\alpha}^{\infty} e^{\frac{-x^{2}}{2}} d x
$$

e

$$
A=\frac{(W / R)(1-\eta)}{\exp \left(\beta m_{x}\right)}
$$

Como $Z^{\prime}$ é uma soma de $k$ variáveis aleatórias, onde o próprio $k$ é

uma variável aleatória, pode ser mostrado que [14] :

$$
\mathrm{E}\left(Z^{\prime}\right)=\mathrm{E}(k) \mathrm{E}\left(v \varepsilon^{\prime}\right)
$$

$$
\operatorname{Var}\left(Z^{\prime}\right)=\mathrm{E}(k) \operatorname{Var}\left(v \varepsilon^{\prime}\right)+\operatorname{Var}(k)\left[\mathrm{E}\left(v \varepsilon^{\prime}\right)\right]^{2}
$$

onde $\varepsilon^{\prime}=\varepsilon \mathrm{e}^{-\beta \mathrm{m}_{\mathrm{x}}}$

Lembrando que $\varepsilon=10^{x / 10}$ onde $\mathrm{x}$ é uma variável aleatória log-normal com média $\mathrm{m}_{x}$ e variância $\sigma_{\mathrm{x}}{ }^{2}$ os valores de $\mathrm{E}$ $\left(v \varepsilon^{\prime}\right)$ e $\operatorname{Var}\left(v \varepsilon^{\prime}\right)$ podem ser facilmente obtidos uma vez que $v$ e $\varepsilon^{\prime}$ são variáveis aleatórias independentes. Para a variável de Bernoulli $v$ temos que $\mathrm{E}(v)=\mathrm{E}\left(v^{2}\right)=\rho \mathrm{e}$, como $k$ é modelada como uma variável aleatória de Poisson, $\mathrm{E}(k)=\operatorname{Var}(k)=\lambda / \mu$. 
A Fig. 1 mostra a probabilidade de queda de enlace para um sistema com parâmetros $W / R=1280, \rho=0,4, m_{x}=$ $7 \mathrm{~dB}$ e $\sigma_{x}=2,5 \mathrm{~dB}$.

Para um sistema de múltiplas células no tradicional modelo hexagonal com anéis de concêntricos, a capacidade é calculada para uma célula central e neste caso devemos considerar também a interferência das outras células. Este cálculo apresenta maior complexidade e normalmente é feito de forma indireta utilizando-se cálculo numérico ou simulação para determinar um fator $f$ representativo da interferência. Este fator pode ser interpretado como o aumento percentual de usuários (ou tráfego) na célula central de referência que produz a mesma interferência produzida pelas outras células. Ou seja, para incluir o efeito das outras células no cálculo de capacidade basta substituir nas equações acima o tráfego $\lambda \mu$ pelo tráfego equivalente $(\lambda / \mu)^{\prime}=(\lambda / \mu)(1+f)$.

\section{EFEITO DO SOFT HANDOFF}

Em um sistema de comunicações móveis CDMA, devido ao reuso universal da frequência, um usuário pode estar em comunicação com duas ou mais estações base ao mesmo tempo. Isto permite que o handoff entre duas estações base seja mais confiável e ocorra sem interrupções na chamada, o que é usualmente conhecido como soft-handoff. No enlace reverso, o soft-handoff garante que cada usuário será, teoricamente, sempre controlado pela estação base com o sinal piloto mais forte, e consequientemente poderá transmitir com um nível de potência menor. Isto significa que o nível da interferência visto por outras células também será menor com o soft-handoff do que seria com o hard-handoff.

Serão apresentados a seguir valores do fator $f$ que foram obtidos em [15] e [16]. Estes valores levam em conta modelos de propagação que incluem a atenuação devido à distância e o efeito do sombreamento, este modelado como uma variável aleatória com distribuição Lognormal. Os valores se encontram nas tabelas 1 e 2 . $\mathrm{Na}$ Tabela 1 onde $n$ é o expoente da atenuação com a distância, observa-se o aumento da interferência à medida que aumenta o desvio padrão do sombreamento ou que diminui expoente da atenuação. Na Tabela 2 nota-se a redução da interferência à medida que aumenta o número $\mathrm{N}_{\mathrm{c}}$ de estações rádio base em soft handoff.

$\mathrm{Na}$ prática, os algoritmos usados não são ideais, e embora o soft-handoff possa realmente diminuir a interferência através de um controle de potência mais apropriado, esta diminuição pode não ser tão marcante quanto a indicada na tabela.

\begin{tabular}{c|ccc}
\hline $\begin{array}{c}\text { Desvio padräo do } \\
\text { sombreamento (dB) }\end{array}$ & & & \\
& $n=3$ & $n=4$ & $n=5$ \\
\hline 0 & 0.77 & 0.44 & 0.30 \\
2 & 0.78 & 0.43 & 0.30 \\
4 & 0.87 & 0.47 & 0.31 \\
6 & 1.09 & 0.56 & 0.36 \\
8 & 1.60 & 0.77 & 0.47 \\
10 & 2.80 & 1.28 & 0.73 \\
12 & 5.93 & 2.62 & 1.42 \\
\hline
\end{tabular}

Tabela 1 - Fator de interferência inter-celular $f(n=3,4$ e 5 com Soft Handoff entre duas células.) (Ref[15])

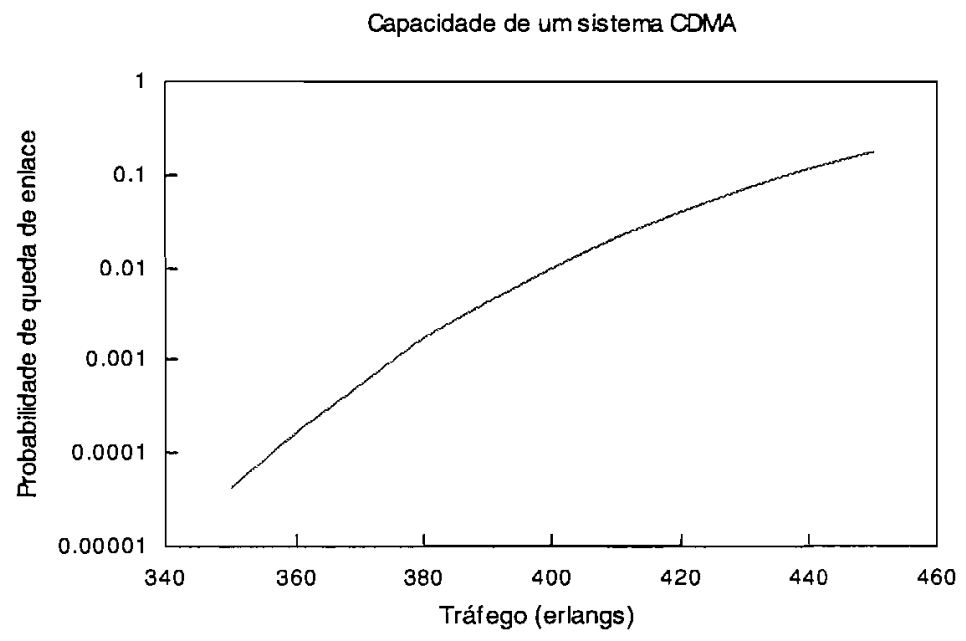

Fig. 1 - Probabilidade de queda de enlace em um sistema com uma única célula. (parâmetros CDMA: W/R=1280; $\rho=0,4 ; \eta=0.1 ; m_{x}=7 \mathrm{~dB} ; \sigma_{x}=2,5 \mathrm{~dB}$ ) 


\section{CAPACIDADE COM LIMITAÇÃO DE CANAIS}

Embora no sistema CDMA IS-95 haja grande disponibilidade de códigos, na prática o número de canais disponíveis depende do número de receptores na estação rádio base. Por outro lado, quanto maior o número de receptores, maior é o custo do sistema, devido ao custo dos modems em si e devido às conexões necessárias com a rede de telefonia fixa. É de se esperar que a partir de um certo valor, um aumento no número de canais represente um ganho insignificante no desempenho do sistema, não compensando um aumento nos custos.

A seguir apresentamos uma extensão da análise da seção anterior incluindo no modelo a possibilidade de bloqueio pela falta de receptores Com isto poderemos determinar o número mínimo de canais que devem estar disponíveis para se manter o grau de serviço de um sistema CDMA.

Consideramos que um usuário poderá ser bloqueado pela não existência de canal disponível e, caso isto não ocorra, ele poderá posteriormente ter o serviço interrompido pela queda do enlace. A ocorrência de um dos dois eventos define a perda da chamada. Denominamos evento $A$ quando o usuário é atendido satisfatoriamente, evento $B$ quando a chamada é bloqueada por falta de canais, e evento $Q$ quando ocorre queda de enlace. Assim, a probabilidade de perda de chamada será a probabilidade do evento $\bar{A}$ dada por

$$
P(\bar{A})=P(B)+P(\bar{B} \cap Q)=P(B)+P(Q \mid \bar{B}) P(\bar{B})
$$

Neste ponto, é importante analisar com mais profundidade a associação entre comportamento da interferência, queda de enlace e perda de chamada. Ao ser combinada com o bloqueio por falta de canais, é necessário que a queda de enlace corresponda à perda da chamada. É claro que uma chamada não é perdida se a interferência ultrapassa o valor máximo permitido só por um intervalo de tempo muito pequeno. Portanto, é necessário caracterizar a perda de chamada pela ocorrência de interferência excessiva com duração acima de um intervalo mínimo de tempo. Esta análise é apresentada no Apêndice, onde resultados de simulações mostram a relação entre a probabilidade de perda de chamada e a probabilidade de queda de enlace. Observa-se que, para um determinado nível de tráfego, a probabilidade de perda de chamada pode ser aproximada pela probabilidade de queda de enlace, desde que 0 intervalo de decisão para descarte da chamada assuma um valor adequado (por exemplo, para um tráfego da ordem de 405 Erlangs, este intervalo estaria em torno de $10 \mathrm{~s}$.)

O novo modelo exige que se calcule a probabilidade de bloqueio usual nos sistemas fixos. Assumindo-se uma taxa de chegada de Poisson de $\lambda$ chamadas/s, um tempo de serviço exponencial com média $1 / \mu \mathrm{s}$, e que caso o usuário seja bloqueado, ele desista da chamada, $P(B)$ pode ser calculado através da fórmula de Erlang-B

$$
P(B)=\frac{(\lambda / \mu)^{N} / N !}{\sum_{i=0}^{N}(\lambda / \mu)^{i} / i !}
$$

onde $N$ é o número de servidores.

O cálculo de $P(Q \mid \bar{B})$ em princípio segue a mesma metodologia apresentada em [1]. Porém agora, com a limitação do número de canais, a distribuição do número de usuários ativos $k$ não será mais de Poisson. A expressão analítica para a média da nova distribuição é calculada sem dificuldade, e é dada por

$$
E(k)=(\lambda / \mu)(1-P(B))
$$

Com relação à variância é empregado um procedimento numérico, a partir do conhecimento das probabilidades dos estados, dadas por

$$
p_{k}=\frac{(\lambda / \mu)^{k} / k !}{\sum_{i=0}^{N}(\lambda / \mu)^{i} / i !}
$$

Estes resultados podem ser substituídos em (10), (11) e (7) para o cálculo da probabilidade de queda de enlace.

Na Fig. 2 mostramos valores das probabilidades de bloqueio por falta de canal, por queda de enlace e por ambos em relação ao número de canais por setor, para intensidades de tráfego diferentes. Foi considerado um sistema de uma única célula, com $W / R=1280, \eta=0,1 \mathrm{e}$ $p=0,4$. O tráfego de 400 Erlangs é o tráfego nominal para uma probabilidade de queda de enlace de $10^{-2}$, e o de 425 Erlangs é nominal para uma probabilidade de $5 \times 10^{-2}$, de acordo com a Fig. 1. Pode ser visto que a probabilidade de queda de enlace aumenta com o número de canais disponíveis, pois desta maneira o número de usuários ativos, na média, também irá aumentar. Podemos observar também que para valores pequenos do número

\begin{tabular}{c|cccc}
\hline $\boldsymbol{\sigma}$ & $\boldsymbol{N}_{\mathrm{c}=1}$ & $\boldsymbol{N}_{\mathrm{c}=\mathbf{2}}$ & $\boldsymbol{N}_{\mathrm{c}=\mathbf{3}}$ & $N_{\mathrm{c}}=\mathbf{4}$ \\
\hline 0 & 0,44 & 0,44 & 0,44 & 0,44 \\
2 & 0,48 & 0,43 & 0,43 & 0,43 \\
4 & 0,67 & 0,47 & 0,45 & 0,45 \\
6 & 1,13 & 0,56 & 0,49 & 0,49 \\
8 & 2,38 & 0,77 & 0,57 & 0,55 \\
10 & 6,17 & 1,28 & 0,75 & 0,66 \\
12 & 19,8 & 2,62 & 1,17 & 0,91 \\
\hline
\end{tabular}

Tabela 2 - Fator de interferência entre células $f$ para um expoente de atenuação com a distância $n=4$ e para $N_{c}$ estações em soft handoff (Ref. [16]) 
de canais disponíveis, pois desta maneira o número de usuários ativos, na média, também irá aumentar. Podemos observar também que para valores pequenos do número ide canais obtemos ganhos consideráveis no desempenho úm um pequeno aumento no número de canais, e estes Ha devidos principalmente a uma redução na forobabilidade de bloqueio. A partir de um certo valor no untanto, esses ganhos são desprezíveis. Para o caso com tráfego de 425 Erlangs em particular podemos perceber îm valor ótimo no desempenho para cerca de 440 canais. Aumentando-se o número de canais iremos observar até mesmo uma piora no desempenho, já que, embora a Grobabilidade de bloqueio seja reduzida, a probabilidade de queda de enlace irá aumentar mais rapidamente.

\section{EFEITO DE FILA DE ESPERA NA CAPACIDADE DO SISTEMA}

is

A retenção das chamadas bloqueadas por falta de canais rem uma fila de espera é um recurso que pode ser explorado para melhorar a eficiência do sistema de a acesso. No sentido de avaliar o potencial deste recurso consideramos nesta seção o efeito de uma fila infinita.
Note-se que neste caso, da mesma forma que na situação de um número ilimitado de canais, não há bloqueio, mas além da probabilidade de queda de enlace devemos também levar em conta como medida de desempenho o tempo de espera médio na fila.

Neste modelo, considerando um sistema com $N$ canais disponiveis, a probabilidade do número total de usuários no sistema ser igual a $n$ é dada por

onde

$$
\begin{gathered}
p_{n}=\left\{\begin{array}{cl}
p_{0} \frac{(\lambda / \mu)^{n}}{n !} & , n \leq N \\
p_{0} \frac{(\lambda / \mu)^{n}}{N ! N^{n-N}} & , n>N
\end{array}\right. \\
p_{0}=\left(\sum_{n=0}^{N} \frac{(\lambda / \mu)^{k}}{n !}+\frac{(\lambda / \mu)^{N}}{N !} \frac{\lambda /(\mu N)}{1-\lambda /(\mu N)}\right)^{-1}
\end{gathered}
$$

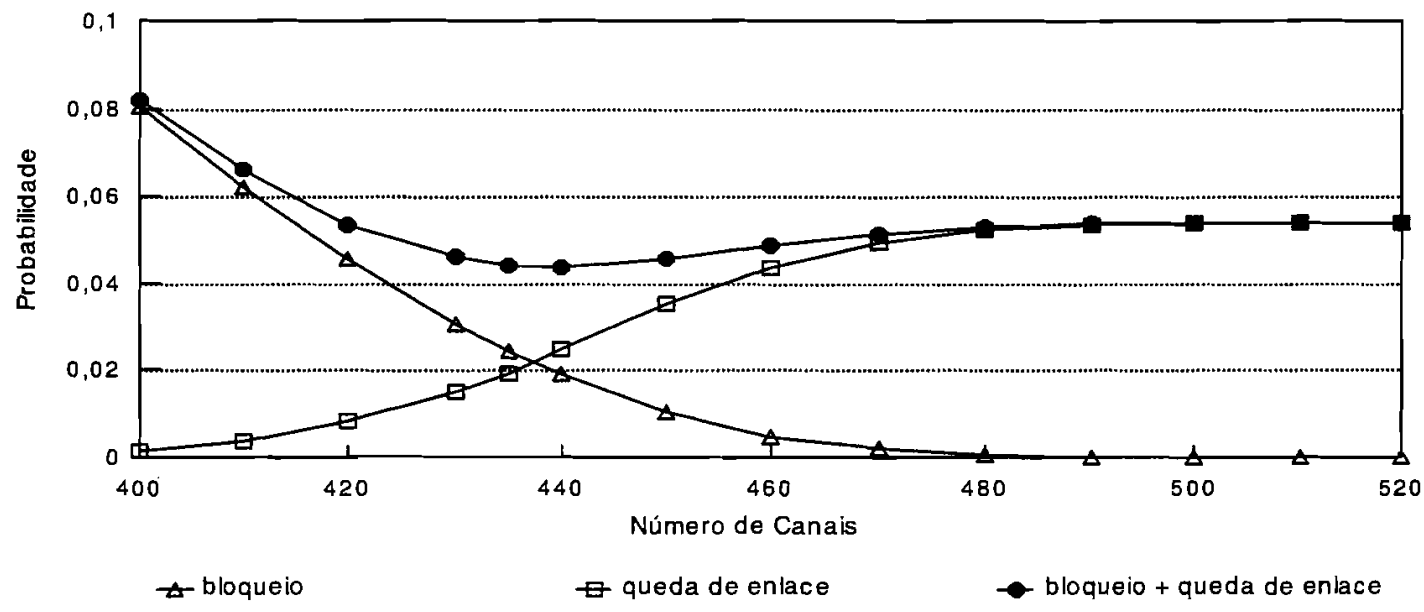

425 Erlangs

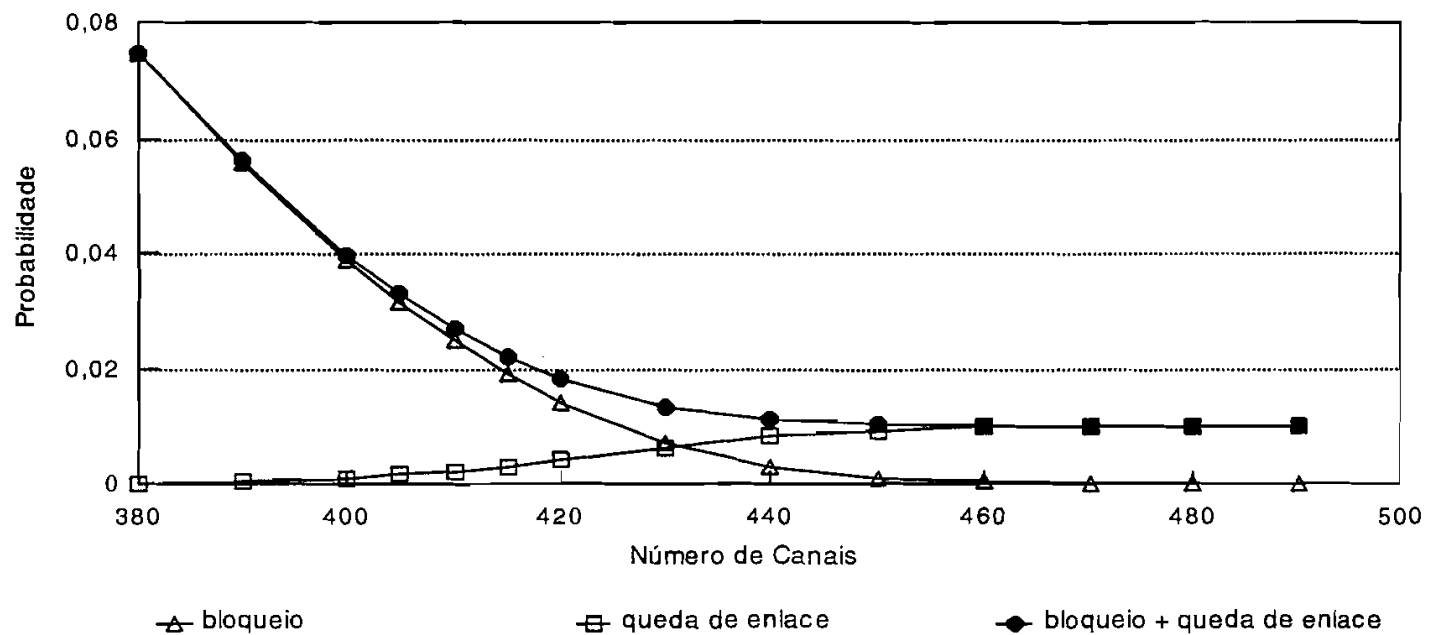

Fig. 2 - Desempenho de um sistema CDMLA com limitação de canais 
Note-se que para que o sistema esteja em equilíbrio, $\lambda(\mu N)<1$.

Para podermos calcular a probabilidade de queda de enlace neste sistema devemos obter os valores de $E[k] \mathrm{e}$ $\operatorname{Var}[k]$, para posterior aplicação nas equações (10), (11) e (7), lembrando que $k$ representa o número apenas de usuários ativos, e que devem levados em consideração para o cálculo da interferência. Com base nas probabilidades de estado acima, dadas por (16) e (17), obtemos

$$
\begin{gathered}
E[k]=\sum_{k=0}^{N} k p_{k}+N \sum_{k=N+1}^{\infty} p_{k}=\lambda / \mu \\
E\left[k^{2}\right]=\sum_{k=0}^{N} k^{2} p_{k}+\sum_{k=N+1}^{\infty} N^{2} p_{k}=\sum_{k=0}^{N} k^{2} p_{k}+\frac{(\lambda / \mu)^{N+1}}{(N-1) !(1-\lambda /(\mu N))} p_{0}
\end{gathered}
$$

A média do tempo de espera na fila $E[T]$ pode ser obtida através da fórmula de Little

$$
E[T]=\frac{E[n]}{\lambda}-\frac{1}{\mu}
$$

onde $E[n]$ é o valor esperado do número total de usuários no sistema, ativos ou não, e este valor pode ser obtido pela seguinte expressão :

$$
E[n]=\sum_{n=0}^{\infty} n p_{n}=p_{0}\left[\sum_{n=0}^{N} n \frac{(\lambda / \mu)^{n}}{n !}+\frac{(\lambda / \mu)^{N+1}}{N !} \frac{N-\lambda / \mu+1}{N(1-\lambda /(\mu N))^{2}}\right]
$$

Na Fig. 3 podemos comparar o desempenho de um sistema com fila de espera com o desempenho de um sistema com bloqueio, definido na seção 3. Podemos ver que a probabilidade de queda de enlace é menor em um sistema sem fila do que em um sistema com fila, já que neste caso os canais estão ocupados uma maior parte do tempo. No entanto, se levarmos em consideração o bloqueio, o sistema com fila apresenta um desempenho bem melhor. Este também apresenta um desempenho melhor inclusive que o valor nominal, obtido para um sistema com infinitos servidores, particularmente para um número pequeno de canais. Isto acontece porque em um sistema com fila o número de usuários interferentes é limitado, o que não acontece no outro sistema. É claro que isto é conseguido às custas de uma espera do usuário, mas podemos ver que este tempo de espera decai rapidamente com o aumento do número de canais. No caso mostrado na Fig. 3, por exemplo, com 400 Erlangs e 420 canais, considerando uma duração média de chamada de $3 \mathrm{~min}$, o tempo médio de espera seria de apenas $2 \mathrm{~s}$.

Podemos concluir pela figura acima que a utilização de uma fila de espera permite uma melhoria no desempenho, com um número reduzido de canais e com um tempo de espera na fila razoável. Este tempo de espera poderia ser utilizado pelo sistema para realizar alguns procedimentos para o estabelecimento da chamada, e desta forma o usuário iria perceber pouca diferença entre um sistema com fila e outro sem fila. Um modelo mais realista deveria considerar que o usuário faria inúmeras tentativas de chamada em caso de bloqueio. Neste caso, o desempenho do sistema sem fila seria ainda pior, tanto em termos de bloqueio quanto interrupção do enlace, e

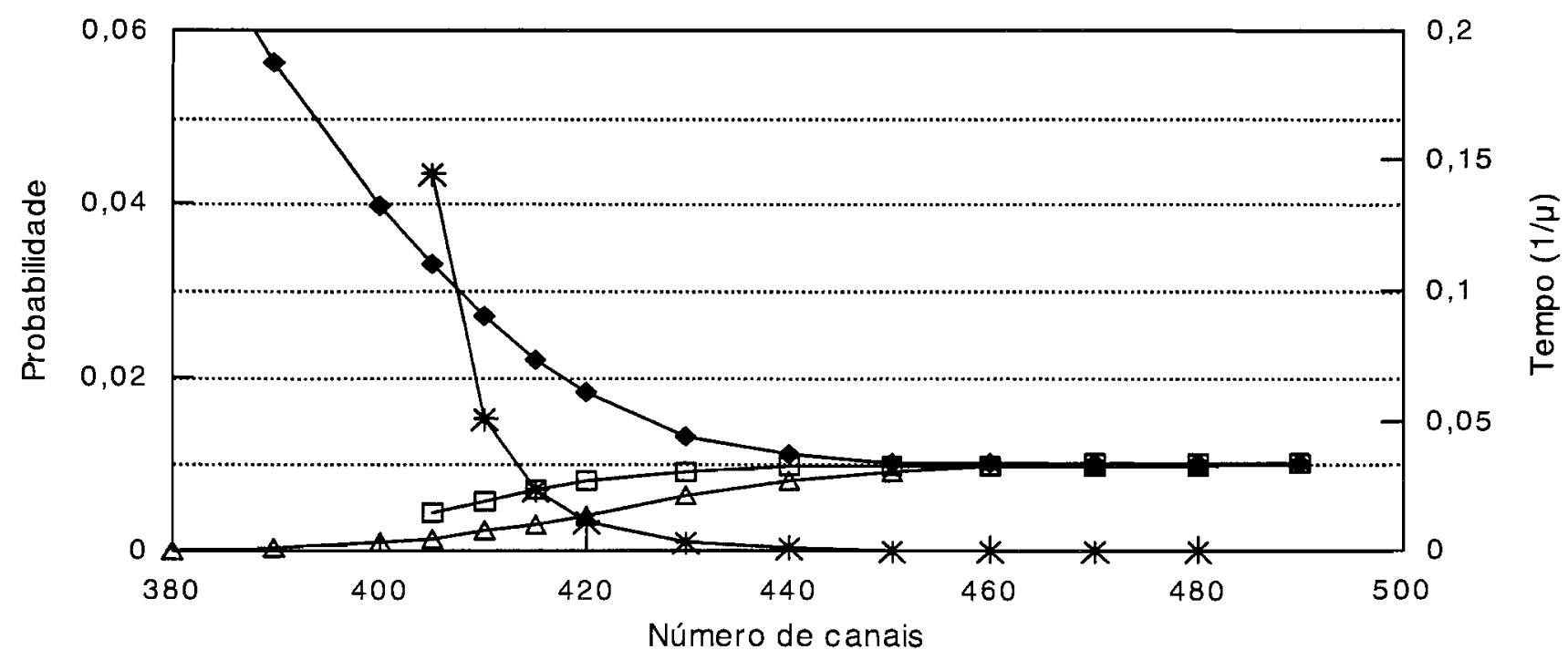

$\Delta P(Q)-$ s/fila

$P(B)+P(Q)-s /$ fila

\#P(Q) - c/ fila

* $\mathrm{E}[\mathrm{T}]$

Fig. 3 - Desempenho de um sistema CDMA com limitação de canais e fila de espera 
conseqüentemente a melhora obtida com o uso de uma fila seria ainda maior. Uma desvantagem do sistema com fila é o aumento na complexidade do sistema, dificultanto a gerência dos recursos do sistema e possivelmente um viabilidade, fazermos provocando aumento no tráfego de sinalização. Ainda é preciso no entanto para um estudo mais detalhado de sua, entre outras coisas, uma melhor avaliação do tamanho ideal da fila, considerando sistemas com filas finitas.

\section{CONCLUSÃO}

Foram analisados alguns aspectos de sistemas CDMA que influem na capacidade de tráfego, mas cujas influências não tinham sido analisadas com detalhe na literatura. Primeiramente verificamos o efeito de uma limitação física do número de canais, devido a um número insuficiente de modems. Com esta limitação, além de considerarmos a probabilidade de queda de enlace como medida de qualidade, devemos levar em conta também a probabilidade de bloqueio devido à falta de canais disponíveis. Através desta análise podemos então estabelecer qual o valor ótimo do número de modems disponíveis em cada célula e assim minimizar os custos. Além disso, sugerimos a implementação de uma fila de espera, e obtemos resultados que comprovam ser possível obter uma melhora no desempenho desta maneira.

Discutimos também o fato de que, para que um usuário perceba alguma degradação no sistema, é necessário que a queda de enlace tenha uma certa duração mínima. Definimos então uma nova medida de qualidade, a probabilidade de perda de chamada, e estudamos a sua relação com a probabilidade de queda de enlace, previamente calculada.

\section{APÊNDICE A \\ RELAÇÃO ENTRE QUEDA DE ENLACE E PERDA DE CHAMADA}

A probabilidade de queda de enlace é definida como a probabilidade de que o nível de interferência esteja acima de um certo limiar em um instante de tempo qualquer, mas, para o usuário, o fato que realmente interessa é se a sua chamada será perdida ou não, devido a esta queda de enlace. Para que o usuário perceba que houve uma queda na qualidade da ligação, é necessário que a interferência permaneça em um nível indesejável durante um certo intervalo de tempo. Além disso, para que o sistema decida que a taxa de erro está inaceitável e a chamada deve ser eliminada, também será necessário um certo intervalo de tempo. Desta forma, a fim de que possamos obter uma medida consistente da probabilidade de bloqueio, devemos calcular, não a probabilidade de queda de enlace, mas a probabilidade de perda de chamada, que é a probabilidade de que a interferência permaneça inaceitável durante um dado intervalo de tempo em uma chamada qualquer.
Uma análise semelhante é feita em [17], onde, através de uma avaliação do número de cruzamentos de nível, são encontradas expressões para a frequência de quedas de enlace de uma certa duração, e para o valor esperado da duração de cada queda de enlace. No entanto em [17] se levam apenas em consideração as variações no tempo devido às mudanças das condições de propagação de um determinado usuário, assumindo um nível de interferência constante. Neste trabalho nós fazemos uma análise diferente, considerando as variações no nível de interferência devido às flutuações no tráfego e à ativação por voz. Além disso, consideramos neste trabalho que a medida de desempenho mais relevante é a probabilidade de perda de chamada, definida no parágrafo anterior.

Consideramos que uma chamada é perdida no instante $t$ se a interferência média $Z_{m}(t)$, calculada em um intervalo de decisão $\tau$, estiver acima de um certo limiar, que é dado por (5). A condição para que não ocorra perda de chamada no instante $t$ é dada então por

$$
Z_{m}(t) \equiv \frac{1}{\tau} \int_{t-\delta}^{i}\left[\sum_{i=1}^{k} v_{i}(\alpha) \varepsilon_{i}(\alpha)+\sum_{j}^{\substack{\text { ouraw } \\ \text { cetiuar }}} \sum_{i=1}^{k} v_{i}^{(j)}(\alpha) \varepsilon_{i}^{(j)}(\alpha)\right] \mathrm{d} \alpha \leq \frac{W}{R}(1-\eta)
$$

A probabilidade de perda de chamada $P_{P}$ em uma chamada de duração $T$ será então a probabilidade de que a condição (A.1) não seja satisfeita para pelo menos um instante $\mathrm{t} \leq \mathrm{T}$. Considerando então que a duração de cada chamada é uma variável aleatória exponencial $\theta$ com média $1 / \mu$, temos que

$$
P_{p}=\int_{0}^{\infty} \mu \mathrm{e}^{-\mu \theta} \operatorname{Pr}\left[Z_{m}(t)>\frac{W}{R}(1-\eta), \text { algum } 0<t \leq T \mid T=\theta\right] \mathrm{d} \theta
$$

Este comportamento da interferência no tempo é extremamente difícil de se determinar analiticamente. Nós decidimos portanto investigar este problema através de uma simulação em computador. Foi simulado um sistema de uma única célula, com um número ilimitado de servidores, onde a chegada de novas chamada é um processo de Poisson com taxa $\lambda$ chamadas por segundo e o tempo de serviço é uma variável aleatória exponencial com média $1 / \mu$ segundos. A razão sinal-interferência $E_{\mathrm{b}}$ $/ I_{0}$ de cada usuário é uma variável aleatória com distribuição log-normal, conforme o modelo usual. Também consideramos que os usuários não estão em movimento, e conseqüentemente a razão sinalinterferência de cada usuário permanece constante durante uma chamada. Em relação à atividade da voz, consideramos que o intervalo de atividade tem uma distribuição exponencial com média $t_{1}$ e cada intervalo de silêncio também é exponencialmente distribuído com média $t_{0}$.

Nas simulações consideramos os valores $1 / \mu=3 \min , t_{1}=$ $1 \mathrm{~s}$ e $t_{0}=1.5 \mathrm{~s}$, o que equivale a um fator de atividade da voz igual a 0,4. Assumimos também os mesmos 


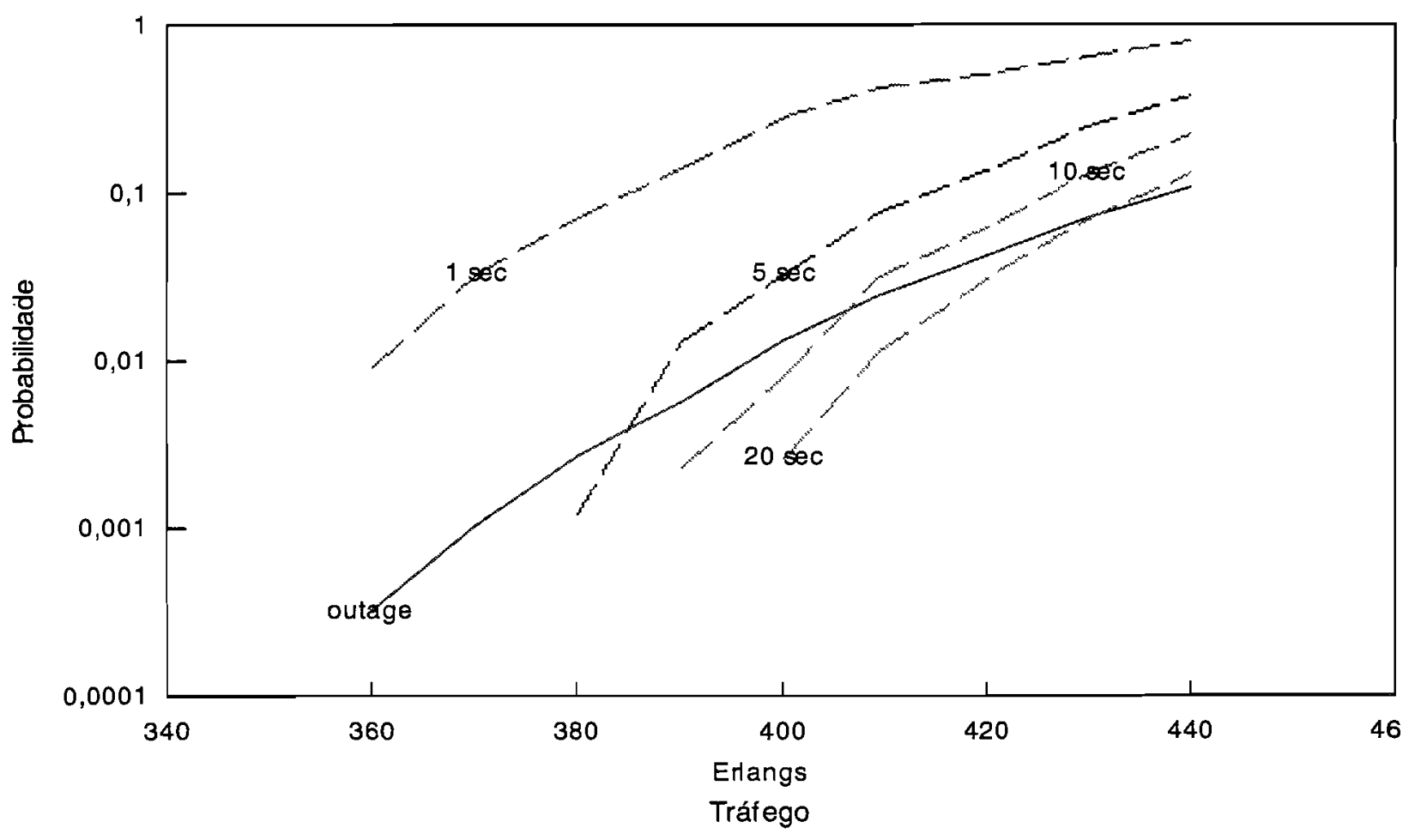

Fig. A.1 - Probabilidade de perda para diferentes intervalos de decisão

parâmetros de sistema considerados na seção 2 , ou seja $W / R=1280, \eta=0,1, m_{x}=7 \mathrm{~dB}$ e $\sigma_{x}=2,5 \mathrm{~dB}$. $O$ cálculo da interferência é feito a intervalos discretos de $0,1 \mathrm{~s}$, e em cada amostragem é calculada a média da interferência nos $\tau$ segundos anteriores.

Na Fig. A.1 podemos ver as curvas da probabilidade de perda em relação ao nível de tráfego para diferentes intervalos de decisão $\tau$. Estas curvas também estão comparadas com uma curva da probabilidade de queda de enlace. Observamos que para intervalos de decisão pequenos a probabilidade de perda é muito maior que a probabilidade de queda de enlace. Isto parece lógico, já que neste caso ocorrerá perda praticamente toda vez que ocorrer queda de enlace, e a probabilidade de perda será aproximadamente a probabilidade de acontecer pelo menos uma queda de enlace durante uma chamada, que é bastante alta. Quando o intervalo de decisão aumenta, a probabilidade de perda se aproxima da probabilidade de queda de enlace, e será eventualmente menor que esta.

A Fig. A.2 mostra a relação entre a probabilidade de perda de chamada e o intervalo de decisão para diversos níveis de tráfego. Para um determinado nível de tráfego podemos determinar por meio desta figura o intervalo de decisão para o qual a probabilidade de perda e a probabilidade de queda de enlace são iguais.

\section{REFERÊNCIAS}

[1] A.J. Viterbi e A.M. Viterbi, "Erlang Capacity of a Power Controlled CDMA System", IEEE Journal on Selected Areas of Communications, pp 892-899, Agosto 1993.

[2] TIA/EIA/IS-95

[3] Gilhousen, Jacobs, Padovani, Viterbi, Weaver e Wheatley III, "On the Capacity of a Cellular CDMA System", IEEE Transactions on Vehicular Technology, pp303-311, Maio 1991.

[4] B.Gudmundson, J.Skold e J.K.Ugland, A Comparison of CDMA and TDMA Systems", Proc. 42nd Vehicular Technology Conference, Maio 1992, pp 732-735

[5] M.R.Heath e P.Newson, "The Capacity of a SpreadSpectrum CDMA System for Cellular Mobile Radio with Consideration of System Imperfections", IEEE Journal on Selected Areas in Communications, Vol. 12, No 4, Maio 1994, pp 683-684

[6] C.Kchao e G.Stüber"Analysis of a direct-sequence spread spectrum cellular radio system", , IEEE Trans. on Comm. Vol 41, No 10, Oct 1993

[7] R.Padovani, "Reverse link performance of IS-95 based cellular systems", IEEE Personal Communications Magazine, Vol. 1, No 3, 1994, pp 28-34 
[8] D.Goeckel e Wayne Stark, "Performance of coded directed-sequence systems with Rake reception in a multipath fading environment", European Transactions on Telecommunications, Vol.6 No 1, Jan-Feb. 1995, pp 41-51

[9] J.S. Lee e L.E. Miller, "On the Erlang Capacity of CDMA Cellular Systems", Globecom 1995, Singapura, pp $1877-1883$

[10]M. Soleimanipour e G.H. Freeman, "A Realistic Approach to the Capacity of Cellular CDMA Systems", Proceedings of the VTC 96, pp 1125-1129.

[11]Q.Shen e W.Krzymien, "The Effect of Fading on the Erlang Capacity of the IS-95 CDMA", Proceedings of the ICC-96, pp 1829-1833

[12]David Everitt, "Traffic Engineering of the Radio Interface for Cellular Mobile Networks", Proceedings of the IEEE, pp 1371-1381, Setembro 94

[13]. A.J. Viterbi, A.M. Viterbi e E. Zehavi, "Performance of Power controlled wideband terrestrial digital communications.", IEEE Transactions on Communications, Vol 41, pp 559-569, Abril 1993

[14] W. Feller, "An Introduction to probability Theory", J. Wiley, Nova Iorque, 1957

[15] A.J. Viterbi, A.M. Viterbi e E. Zehavi, "Other-cell interference in cellular power-controlled CDMA.", IEEE Transactions on Communications, Vol 42, pp 1501-1504, Abril 1994
[16] Viterbi, A.J., "CDMA - Principles of Spread Spectrum Communication", Addison-Wesley, 1995

[17]N.B. Mandayan, P. Chen e J.M. Holtzman, "Minimum Duration outage for Cellular Systems : A Level Crossing Analysis", Proceedings of the VTC 96, pp 879-883

André Noll Barreto - formou-se em Engenharia Elétrica em 1994 pela Pontifícia Universidade Católica do Rio de Janeiro e obteve o título de Mestre em Ciências em Engenharia Elétrica pela mesma Universidade em 1996. Participou nos últimos anos como bolsista de iniciação científica e pesquisador assistente, de diversos trabalhos de pesquisa no CETUC - Centro de Estudos em Telecomunicações da PUC/Rio, na área de sistemas de transmissão digital e comunicação celular. Atualmente está no Programa de Doutorado da Universidade de Dresden - Alemanha.

João Célio Barros Brandão - Engenheiro Eletricista pela Universidade de Juiz de Fora (1969) e Mestre em Ciências em Engenharia Elétrica pela PUC/Rio (1973). Professor e pesquisador do CETUC - Centro de Estudos em Telecomunicações da PUC/Rio - desde 1971 onde é atualmente Professor Associado e Vice-Diretor. Em 1987 e 1988 trabalhou no COMSAT Laboratories, U.S.A. na área de comunicações via satélite. No CETUC tem participado, como pesquisador e coordenador, de diversos projetos de pesquisa, em convênio com o Centro de Pesquisa da Telebrás, nas áreas de interferência entre sistemas de comunicações, simulação de sistemas de transmissão digital e sistemas móveis celulares digitais. $\dot{E}$ membro da Comissão Brasileira de Estudos em Radio

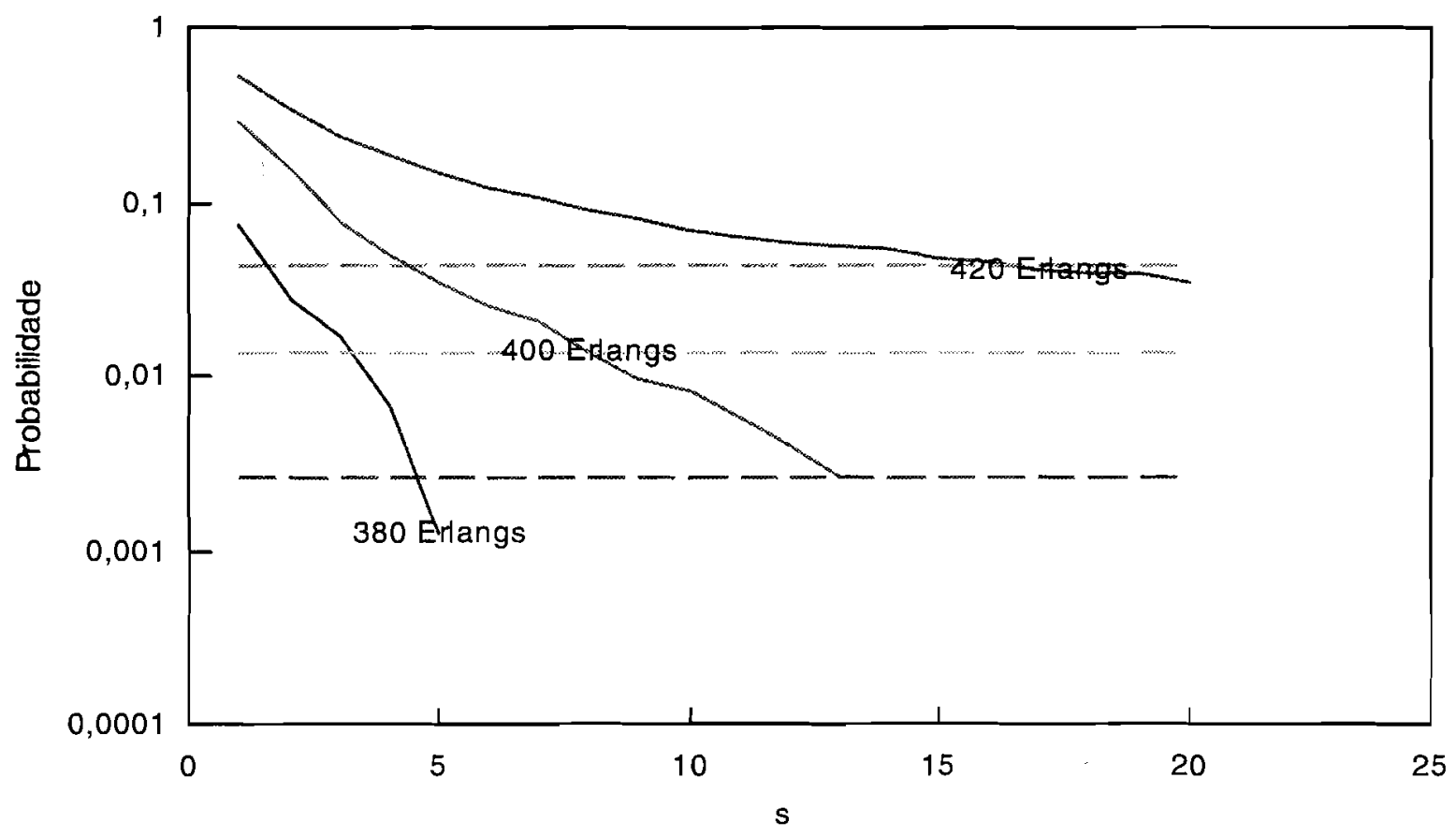

Intervalo de decisão para perda

Fig. A.2 - Probabilidade de perda x Intervalo de decisão

( Linha horizontal pontilhada : probabilidade de queda de enlace) 
Comunicações do Ministério das Comunicações. É sócio fundador da Sociedade Brasileira de Telecomunicações onde foi Editor do Boletim informativo. 